\title{
Experimental study of the penetration of oxygen and deuterium atoms into porous water ice
}

\author{
M. Minissale ${ }^{1}$, T. Nguyen ${ }^{2}$, and F. Dulieu ${ }^{2}$ \\ 1 Aix-Marseille Université, CNRS, PIIM, Marseille, France \\ e-mail: marco.minissale@univ-amu.fr \\ ${ }^{2}$ LERMA, Université de Cergy Pontoise, Sorbonne Université, PSL Research University, Observatoire de Paris, UMR 8112 CNRS, \\ 5 mail Gay Lussac, 95000 Cergy Pontoise, France \\ e-mail: francois.dulieu@obspm.fr
}

Received 11 July 2018 / Accepted 24 December 2018

\begin{abstract}
Context. Many interstellar molecules are thought to form on dust grains. In particular, hydrogenation is one of the major mechanisms of the formation of mantle ice. To date it is not clear if $\mathrm{H}$ atoms can penetrate the bulk of the ice mantle or if it only has chemical activity on the accessible surface of grains.

Aims. We wish to study the efficiency of atoms deposited on the outer surface of the amorphous solid water to penetrate into the ice bulk.

Methods. NO molecules react with $\mathrm{O}$ and $\mathrm{H}$ atoms. They are easily detected by infrared (IR) spectroscopy. These two properties make this molecule an ideal chemical tracer for the penetration of $\mathrm{O}$ and $\mathrm{H}$ atoms through water ice. In our experiments we first deposited a NO undercoat and covered this layer (at $40 \mathrm{~K}$ ) with a variable amount of water ice. Then, we exposed this undercoat to D (10 K) or $\mathrm{O}(40 \mathrm{~K})$ atoms, and we followed the NO consumption and the products that appeared via IR signatures, and we finally analyzed the desorption of all species through a temperature-programmed desorption technique. We experimentally characterize the accessible surface of the ice and provide a model to interpret quantitatively our measurements.

Results. Water ice limits the destruction of tracer NO molecules. The thicker the ice, the more $\mathrm{NO}$ remains unreacted. $\mathrm{H}$ and $\mathrm{O}$ atoms lead to the same amount of NO consumption, pointing out that access to reactants for these two different atoms is identical. We discuss different possible scenarios of NO localization (in and/or on the ice) and determine how this affects our observables (IR data and desorption profiles).

Conclusions. In our experimental conditions, it is not possible to measure any atom penetration through the bulk of the ice. The surface diffusion followed by reaction with $\mathrm{NO}$ or by self-reaction (i.e., $\mathrm{H}+\mathrm{H} \longrightarrow \mathrm{H}_{2}$ ) is faster than bulk diffusion. We propose lower limit values for penetration barriers. Therefore the building of astrophysical ice mantles should be mostly driven by surface reactivity.
\end{abstract}

Key words. ISM: molecules - molecular processes - astrochemistry - diffusion - atomic processes - solid state: volatile

\section{Introduction}

The formation of certain molecules (such as $\mathrm{H}_{2}, \mathrm{H}_{2} \mathrm{O}$ or $\mathrm{CO}_{2}$ ) in different astrophysical environments is possible thanks to the catalytic effect of dust grains (e.g., Anders et al. 1974; Pirronello et al. 1999; Cazaux et al. 2010, and references therein). In dense clouds, where the UV photons flux is rapidly attenuated, atoms and molecules accumulate on dust grains and react to form increasingly complex molecules (Caselli \& Ceccarelli 2012). The diffusion of the lightest species, in particular hydrogen and oxygen atoms, is often considered as the trigger of chemical reactions that are at the base of the growth of these molecular mantles and differ in composition from the gaseous phase. It was shown very early (Tielens \& Hagen 1982) that grain chemistry was mainly limited by diffusion (Cuppen et al. 2017). Experimentally it has been demonstrated that $\mathrm{H}, \mathrm{O}$, and $\mathrm{N}$ atoms can effectively diffuse on the surface of iced grains at low temperature ( $10 \mathrm{~K}$; Matar et al. 2008; Watanabe et al. 2010; Hama et al. 2012; Minissale et al. 2013a, 2016; Congiu et al. 2014; Wakelam et al. 2017).

This type of catalysis dominated by surface diffusion is very effective. The synthesis of methanol from the CO-hydrogenation is an emblematic example, since this molecule is abundantly produced and detected (e.g., Kristensen et al. 2010; Ceccarelli et al. 2017) despite the reaction barriers (Hiraoka et al. 2002; Hidaka et al. 2007; Rimola et al. 2014). It is not yet well understood if once the molecular mantle is built as a consequence of surface reactions, this mantle can evolve because of reactions in its interior. It is known for example that UV penetrates many molecular layers (e.g., $1 \mu \mathrm{m}$ at $163 \mathrm{~nm}$, Orzechowska et al. 2007) creating radicals in the inner layers of the mantle leading to chemical recomposition. It is therefore necessary to understand what is the diffusion in the bulk. Several studies have focused on the experimental determination of bulk diffusion rates of molecules such as $\mathrm{NH}_{3}, \mathrm{CO}$, and $\mathrm{CO}_{2}$ (Mispelaer et al. 2013; Lauck et al. 2015; He et al. 2017; Cooke et al. 2018; Ghesquière et al. 2018) on relevant astrophysical ice, but little is known about the bulk diffusion of atoms. However, it is very important to determine if and how the diffusion of atoms takes place in ice, since some increase of the mobility may be expected for atoms, especially H. An efficient diffusion in bulk can indeed allow a rich chemistry in the ice of dense clouds thus favoring the dynamic and temporal evolution of the ice in its whole thickness. A lack of bulk mobility, in the absence of subsequent processing, 
causes the ice composition to be governed by the surface diffusion and the composition of the accreted gas until the ice is heated or processed.

Water ice is the main compound of astrophysical ices (Boogert et al. 2015), but it is a multiform matrix. In astrophysical environments, water ice can be crystalline or amorphous, referred to as amorphous solid water (ASW), but in dense clouds water iced is thought to be amorphous because some energy processes must take place before it crystallizes (Papoular 2005; Palumbo 2006). Furthermore amorphous ice can be porous or compact: if it is deposited at low temperature $(<70 \mathrm{~K})$, it is porous (Stevenson et al. 1999), but it can be recompacted by ionic or UV irradiation (Palumbo et al. 2010) or by simple chemical activity such as hydrogen recombination (Accolla et al. 2011). Finally, if the ice forms directly on the dust grains, it is amorphous and compact (Oba et al. 2009; Accolla et al. 2013). Of course, the diffusion rates of species in the bulk of ice depend on the ice morphology (amorphous or crystalline) and its degree of porosity.

The reactivity of atoms with another compound inserted in the ice (used as a chemical tracer) can be used to experimentally measure atomic diffusion on/in the water ice. If the reaction is barrier-free, the disappearance of the reactive species allows us to infer the access of the atoms to the reactants, and hence their mobility. This is the method we used in Matar et al. (2008) using $\mathrm{O}_{2}$ consumption as a marker for surface diffusion of $\mathrm{D}$. If we now deposit water ice on top of a reactive partner, by examining the consumption and formation of species according to the thickness of the water ice deposited on top of it, we must be able to deduce the penetration of atoms into the ice. In the present case we use NO, which has no reaction barrier with $\mathrm{H}$ (Congiu et al. 2012) and $\mathrm{O}$ (Minissale et al. 2014b) and is detectable in the IR, contrary to $\mathrm{O}_{2}$. However this approach presents certain constraints from an experimental point of view. Compact water ice (amorphous or crystalline) can only grow at sample temperatures above $100 \mathrm{~K}$. Such temperatures are sufficiently high to induce immediate desorption of the "marker", the reactive compound NO. We are therefore limited by the desorption temperature of the chemical marker (i.e., $\sim 40 \mathrm{~K}$ for NO). This temperature does not allow for the formation of compact ice. The amorphous and porous water ice is what can be studied by this method.

There is a general consensus that water ice in dark clouds is mainly amorphous, but the nature of its porosity degree still remains poorly known (Öberg et al. 2009, 2011; Accolla et al. 2013). For this reason we decided to perform a first experimental study of volumetric diffusion rates of $\mathrm{H}$ and $\mathrm{O}$ atoms on porous ASW.

Strictly speaking, diffusion on porous ice is both a surface diffusion and a bulk diffusion. Figure 1 depicts this situation. There is surface diffusion on the accessible surface of the pores of the ice, which can be located "inside the ice" and therefore this surface diffusion allows the ice to be swept within its volume by the diffusive atoms. This scenario is sometimes called percolation. Bulk diffusion may occur, but refers to the crossing of a compact/closed ice layer. In particular, if closed pores are present, the atoms must penetrate through the wall of the pores before possibly continuing the surface diffusion on the inner surface of the closed pore.

For the rest of the article we call "surface diffusion" the diffusion on both inner and outer surfaces. Thus, the atoms can penetrate in the depth of the ice through surface diffusion, which may appear misleading. This is because of the complex structure of the ice that we used. The bulk diffusion corresponds to the diffusion that requires crossing a water layer.

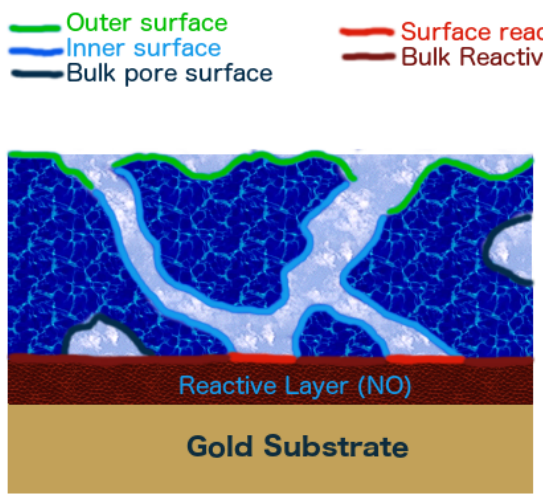

Fig. 1. Schematic drawing of the porous amorphous ice grown on top of a NO layer deposited on a gold surface. The outer surface (in green) is the surface directly accessible from the gas phase (with a normal incidence). The inner surface (in blue) is the surface accessible via surface diffusion. Bulk diffusion is required to access closed pores (dark blue). The reactive layer can be accessed via surface diffusion (both inner and outer surfaces) along the cracks or pores. This case is represented in light red. Part of the reactive layer that required bulk diffusion is drawn in dark red.

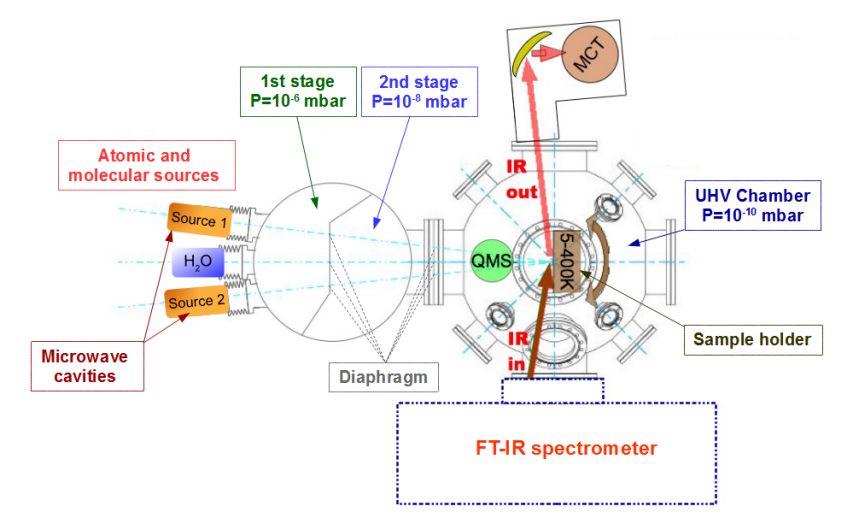

Fig. 2. Schematic top view of the Venus setup and the FT-RAIRS facility.

Our article is organized as follows: the following section describes the experimental setup and ice characterization. In Sect. 3 we presents the experimental results. In Sect. 4, we propose a model of rate equations that simulates our experiments. In the last section, we discuss the main conclusions and astrophysical implications of this study.

\section{Experimental methods}

\subsection{Experimental setup}

The experiments were conducted with the VENUS setup located at LERMA-Cergy lab in Cergy-Pontoise, France. It consists of an ultra high vacuum (UHV) main chamber with a base pressure of $10^{-10}$ mbar. A golden mirror, placed at the center of UHV chamber, is held at temperatures between 8 and $400 \mathrm{~K}$. The temperature is controlled by a calibrated silicon-diode sensor clamped on the sample holder. Atomic species are directed toward the sample through three diaphragms located a double vacuum chamber to ensure a good differential pumping and keep the pressure in the main chamber very low (less than $5 \times$ $10^{-10}$ mbar, during multibeams exposure). The scheme of the setup is shown in Fig. 2. 

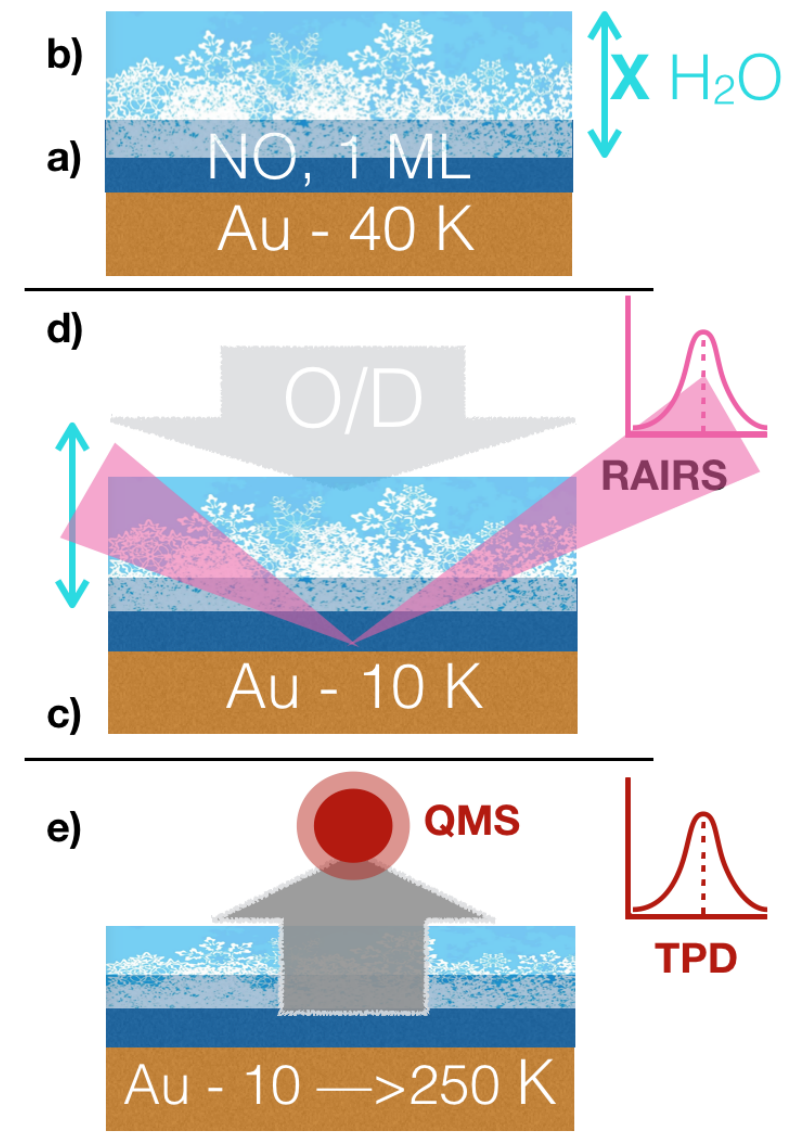

Fig. 3. Scheme of the experimental protocol. Panel $a$ : deposition of $1 \mathrm{~mL}$ of $\mathrm{NO}$ at $40 \mathrm{~K}$. Panel $b$ : deposition of $\mathrm{X} \mathrm{mL}$ of $\mathrm{H}_{2} \mathrm{O}$ at $40 \mathrm{~K}$. Panel c: cool down at $10 \mathrm{~K}$ in the case of D-exposure experiments. Panel d: deposition of $\mathrm{D}$ atoms at $10 \mathrm{~K}$ or of $\mathrm{O}$ atoms at $40 \mathrm{~K}$, recording continuously IR spectra. Panel $e$ : TPD profiles of the species of interest.

Adsorbates and products were probed in situ through a Fourier Transform Reflection Absorption Infrared Spectrometer (FT-RAIRS), and a quadrupole mass spectrometer (QMS) used for measuring the beams fluxes and beams compositions and for performing the temperature-programmed desorption (TPD) experiments.

All the experiments were performed following these steps:

(a) NO deposition. One monolayer $\left(\mathrm{mL}=10^{15}\right.$ molecules $\left.\mathrm{cm}^{-2}\right)$ of NO on gold sample held at $40 \mathrm{~K}$.

(b) $\mathrm{H}_{2} \mathrm{O}$ deposition. $\mathrm{X} \mathrm{mL}$ of $\mathrm{H}_{2} \mathrm{O}$ on top of $\mathrm{NO}$ ice at $40 \mathrm{~K}$ $(X=0,2,3,6$, and $10 \mathrm{~mL})$.

(c) Sample temperature. Cool down to $10 \mathrm{~K}$ in the case of $\mathrm{D}$ atoms study. The surface is kept at $40 \mathrm{~K}$ for $\mathrm{O}$ study.

(d) Beam exposure. $\mathrm{NO}-\mathrm{H}_{2} \mathrm{O}$ ice are exposed to a D- or O-atom beam. RAIRS spectra recorded during exposure.

(e) TPD performed after $\mathrm{D}$ or $\mathrm{O}$ exposure.

This protocol is schematized in Fig. 3.

Deuterium is used in place of hydrogen to increase the signal-to-noise ratio $(\mathrm{S} / \mathrm{N})$. In the case of $\mathrm{D}$ atoms exposure, we held at $10 \mathrm{~K}$ after the same procedure of ice formation at $40 \mathrm{~K}$. The ice is not supposed to change upon cooling, but can change the morphology if heated. During the $\mathrm{O}$ atoms exposure, $\mathrm{NO}-\mathrm{H}_{2} \mathrm{O}$ ice is held at $40 \mathrm{~K}$ to enhance $\mathrm{O}_{2}$ desorption and prevent an efficient formation of $\mathrm{O}_{3}$ (Minissale et al. 2014a). These temperatures are chosen so that the diffusion of atoms is fast enough and their desorption is negligible. Both $\mathrm{D}$ and $\mathrm{O}$ should be in the thermal diffusion regimes in our experimental conditions (Hama et al. 2012; Senevirathne et al. 2017; Congiu et al. 2014).

Atomic species, $\mathrm{D}$ and $\mathrm{O}$ atoms, were generated by dissociating $\mathrm{D}_{2}$ and $\mathrm{O}_{2}$ molecules in a quartz tube placed within a Surfatron cavity, which can deliver a maximum microwave power of $200 \mathrm{~W}$ at $2.45 \mathrm{GHz}$. Atoms and undissociated molecules were cooled and instantaneously thermalized upon surface impact with the walls of the quartz tube. Deuterium and oxygen beams were free of electronic excited atoms (Congiu et al. 2009; Minissale et al. 2015). The dissociation efficiency of $\mathrm{D}_{2}$ and $\mathrm{O}_{2}$ was $\tau=50 \pm 5 \%$, where $\tau$ represents the percentage of dissociated molecules. The value $\tau$ defines the atom/molecule ratio in the beam. For example if $\tau$ is 0.5 , every ten molecules we have ten atoms and five undissociated molecules.

We calibrated the molecular beam as described in Amiaud et al. (2007) and Noble et al. (2012). The first monolayer of NO was reached after an exposure time of about $15 \mathrm{~min}$, which give a flux of $\phi_{\mathrm{NO}}=(1.0 \pm 0.3) \times 10^{12}$ molecules $\mathrm{cm}^{-2} \mathrm{~s}^{-1}$. The $\mathrm{D}_{2}$ and $\mathrm{O}_{2}$ molecular fluxes were $\phi_{\mathrm{D}_{2}, \mathrm{O}_{2}}=(1.05 \pm 0.3) \times$ $10^{12}$ molecules $\mathrm{cm}^{-2} \mathrm{~s}^{-1}$ while $\mathrm{D}$ and $\mathrm{O}$ atoms fluxes were $\phi_{\mathrm{D}, \mathrm{O}}=(2.1 \pm 0.3) \times 10^{12}$ atoms $\mathrm{cm}^{-2} \mathrm{~s}^{-1}$.

Adsorbates and products were probed continuously through FT-RAIRS during atoms exposure. TPD was performed after D or $\mathrm{O}$ atoms exposure and the sample was heated up to 250 or $190 \mathrm{~K}$, respectively. Those temperatures allow the desorption of $\mathrm{NO}-\mathrm{H}_{2} \mathrm{O}$ ice and of newly formed molecules $\left(\mathrm{ND}_{2} \mathrm{OD}\right.$ and $\mathrm{N}_{2} \mathrm{O}$ in the case of $\mathrm{D}$, and $\mathrm{NO}_{2}$ in the case of $\mathrm{O}$ ).

We use the $\mathrm{mL}$ unit, which corresponds to $10^{15}$ molecules $\mathrm{cm}^{-2}$ exposed to the surface. For a typical solid substrate, like crystalline water ice, it also corresponds to the number of adsorption sites (NAS). But in the case of a reactive species, like $\mathrm{O}$ atoms, exposing $1 \mathrm{~mL}$ does not mean that one layer of $\mathrm{O}$ atoms is deposited, since $\mathrm{O}$ atoms diffuse and react quickly. If only $\mathrm{O}_{2}$ is formed (like in the case of exposure on a nonreactive surface held at $30 \mathrm{~K}$ ), and if the desorption (thermally or chemically induced) is neglected, it would lead to $50 \%$ of occupancy of the surface binding sites by $\mathrm{O}_{2}$ molecules, because two $\mathrm{O}$ atoms are recombining into one $\mathrm{O}_{2}$ molecule. Moreover, in the case of light species, such as $\mathrm{H}, \mathrm{H}_{2}, \mathrm{D}$, the sticking coefficient is far to be unity (Chaabouni et al. 2012), so the exposed dose does not correspond to the number of molecules adsorbed on the surface. Finally, when the surface is porous or in presence of clusters, the NAS can greatly vary, and in general, even if the sticking coefficient is unity, $1 \mathrm{~mL}$ of deposition is not sufficient to cover the full accessible surface, and therefore the saturation of the surface can occur after few $\mathrm{mL}$ of exposure.

\subsection{Evaluation of the number of adsorption sites with the water ice thickness}

Water ice deposited at $40 \mathrm{~K}$ is porous (Stevenson et al. 1999). The presence of cavity and pores on the surface increases the NAS and thus the effective surface. We studied how the effective surface seen by the atoms evolves as a function of water ice layer. We used a standard King and Wells method (King \& Wells 1972) by depositing $D_{2}$ as a function of water ice thickness. We monitor the partial pressure of $\mathrm{D}_{2}$ in the chamber while a $\mathrm{D}_{2}$ beam is sent to the surface held at $10 \mathrm{~K}$. Typical measured curves can be seen in Fig. 3 of Amiaud et al. (2007). For each ice substrate there is a specific saturation time that scales to the NAS. The method does not measure directly the NAS since the $\mathrm{D}_{2}$ saturation of compact ASW occurs at $0.4 \mathrm{~mL}$ (of exposure) at $10 \mathrm{~K}$, which corresponds to an occupancy of 


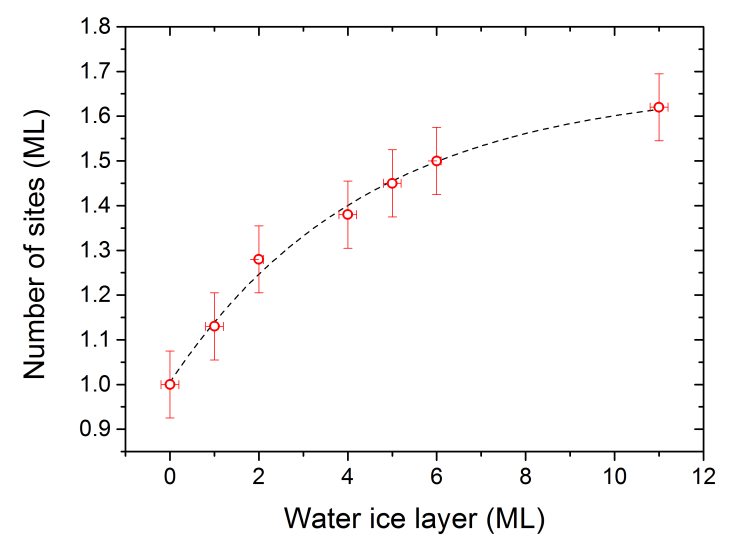

Fig. 4. Number of adsorption sites expressed in $\mathrm{mL}$ as a function of water ice thickness, derived from a King and Wells method. The initial surface is a compact ASW of $15 \mathrm{~mL}$ thickness grown at $110 \mathrm{~K}$ covered with the addition of extra layers of porous water.

$\sim 15 \%$ of the surface sites taking into account the sticking coefficient (Amiaud et al. 2007). However, the assumption that the saturation time of $\mathrm{D}_{2}$ scales to the NAS is quantitatively verified with one experimental direct measurement at $5 \mathrm{~mL}$ of overlayers of $\mathrm{H}_{2} \mathrm{O}$, using $\mathrm{N}_{2}$ and $\mathrm{CO}$ as direct probes (Nguyen et al. 2018).

The results are shown in Fig. 4. Without addition of amorphous porous water, the surface is considered as planar. The initial surface is a compact ASW of $15 \mathrm{~mL}$ thickness grown at $110 \mathrm{~K}$. It is by definition equal to $1 \mathrm{~mL}$. With the addition of extra layers of porous water, the saturation time increases, which corresponds to an increase of the NAS. The effective surface reaches 1.6 for $11 \mathrm{~mL}$ of extra water ice deposited at $40 \mathrm{~K}$. In order to take into account the evolution of NAS in the model presented in Sect. 4, we fitted the experimental results with the following exponential law

$\mathrm{NAS}=1.67-0.67 \cdot e^{-\frac{\mathrm{H}_{2} \mathrm{O}}{4.43}}$

We stress that this is an empirical law and it is far from being a general law to describe the evolution of NAS as a function of water ice thickness. It is only valid in our specific experimental conditions.

\section{Experimental results}

\subsection{Oxygenation of NO ices}

The diffusion of oxygen in ASW is studied using nitric oxide as a reactive marker. Oxygenation of $\mathrm{NO}$ can occur through $\mathrm{NO}$ reactions with the three oxygen allotropes $\mathrm{O}_{x}\left(\mathrm{O}, \mathrm{O}_{2}\right.$, and $\left.\mathrm{O}_{3}\right)$. Solid state reactions generally have two limiting factors, the reagent diffusion barrier and the reaction barrier. As the $\mathrm{NO}+\mathrm{O}$ reaction is barrier-free, it represents a system suitable for the study of $\mathrm{O}$ diffusion. We note that the presence of atomic oxygen on a surface easily leads to the formation of diatomic oxygen and ozone therefore, reactions $\mathrm{NO}+\mathrm{O}_{2}$ and $\mathrm{NO}+\mathrm{O}_{3}$ could occur in solid phase and would make the interpretation of experimental results more difficult. To avoid this problem, the presence of diatomic oxygen and ozone can be limited by performing experiments at a sample temperature of $40 \mathrm{~K}$. At this temperature, $\mathrm{O}_{2}$ is efficaciously released in the gas phase and, therefore, $\mathrm{O}_{3}$ cannot be effectively formed in the solid phase (Minissale et al. 2014a).

Figure 5 shows typical IR spectra obtained by exposing $1 \mathrm{~mL}$ of $\mathrm{NO}$ held at $40 \mathrm{~K}$ to $\mathrm{O}$ atoms. The red spectrum is taken before

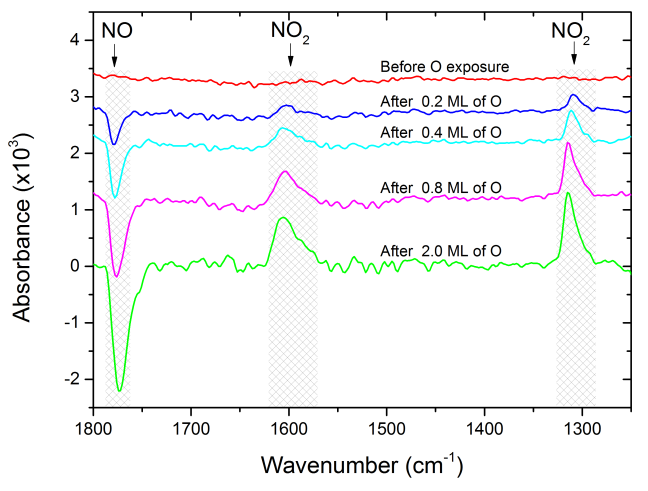

Fig. 5. Infrared spectra obtained by exposing to $1 \mathrm{~mL}$ of $\mathrm{NO}$ held at $40 \mathrm{~K}$ to different amount of $\mathrm{O}$ atoms: $0,0.2,0.4,0.8$, and $2.0 \mathrm{~mL}$. The red curve does not present any IR signature since it has been considered as the reference spectrum. Curves are offset for clarity.

O-atom exposure. This curve does not present any IR signature since it has been taken as reference spectrum. The other spectra are taken after $0.2,0.4,0.8$, and $2.0 \mathrm{~mL}$ of $\mathrm{O}$-atom exposure, respectively. We can assign three main signatures: the NO dimer at $1770 \mathrm{~cm}^{-1}$ and the $\mathrm{NO}_{2}$ at 1605 and $1315 \mathrm{~cm}^{-1}$. From the decreasing of $\mathrm{NO}$ peak as a function of $\mathrm{O}$-atom exposure, we evaluate the kinetics of NO consumption. Similarly, by integrating the increasing of $\mathrm{NO}_{2}$ signatures, we evaluate the formation rate of $\mathrm{NO}_{2}$.

The kinetics of $\mathrm{NO}$ consumption and $\mathrm{NO}_{2}$ formation are shown in Fig. 6 as a function of O-atom exposure for different thickness of $\mathrm{H}_{2} \mathrm{O}$ ice $(0,2,3,6$, and $10 \mathrm{~mL})$. Each curve shows a decrease of the reagent ( $\mathrm{NO}$, top panel) correlated with an increase of one of the product $\left(\mathrm{NO}_{2}\right.$, bottom panel). As expected for a barrierless reaction the kinetics reach a steady-state plateau after an exposure about equal to one $\mathrm{mL}$, which corresponds to a perfect stochastic reaction.

As evident from experimental data in Fig. 6, the level of the steady state of $\mathrm{NO}$ consumption (or $\mathrm{NO}_{2}$ formation) depends on the thickness of water ice. When no water ice is deposited on top of $\mathrm{NO}, 1 \mathrm{~mL}$ of oxygen atoms is able to react with all the NO previously grown $(\sim 1 \mathrm{~mL})$. Two $\mathrm{mL}$ of porous water ice are able to prevent a complete consumption of NO ice since less than $0.7 \mathrm{~mL}$ are consumed. For $10 \mathrm{~mL}$ of water ice, only $0.1 \mathrm{~mL}$ of NO reacts with $\mathrm{O}$ atoms. We note that once the steady-state plateau is reached, there is no measurable slower increase within our experimental uncertainties

As explained in the experimental procedure, the NO ice deposit is followed by a $\mathrm{H}_{2} \mathrm{O}$ deposit on the $\mathrm{NO}$ ice. However, because of the porous structure of the water ice and diffusion, NO molecules may be present in an "uncovered" adsorption site after water ice deposition.

The following two populations of NO can therefore be considered:

- covered NO, accessible only by pure bulk diffusion of $\mathrm{O}$ atoms (hereinafter $\mathrm{NO}_{b}$ )

- uncovered $\mathrm{NO}$, present on the surface or in the pores of water and accessible by surface diffusion of $\mathrm{O}$ atoms (hereinafter $\mathrm{NO}_{s}$ ).

In the $\mathrm{NO}_{s}$ population, there are two different subcategories, which could be called "outer surface", and "inner accessible surface". They are depicted in green and blue, respectively, in Fig. 1. The first category corresponds to NO located on the outer surface, and thus can interact directly with the gas. Even though water ice is deposited on top of the NO layer, we cannot neglect 


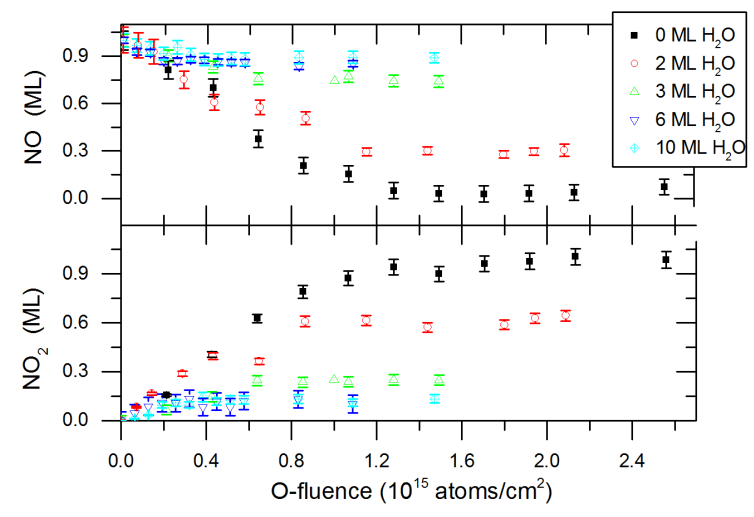

Fig. 6. Kinetics of $\mathrm{NO}$ consumption and $\mathrm{NO}_{2}$ formation obtained by integrating IR peaks of $\mathrm{NO}$ and $\mathrm{NO}_{2}$ as a function of O-atom fluence for five different thickness of water ices $(0,2,3,6$, and $10 \mathrm{~mL})$ held at $40 \mathrm{~K}$.

the possibility for NO to diffuse during the ice building at $40 \mathrm{~K}$. The second category of $\mathrm{NO}_{s}$ corresponds to $\mathrm{NO}$ located in a pore, and it requires surface diffusion of $\mathrm{O}$ (or D) to react. However NO molecules organize themselves as dimers (Minissale et al. 2013b, 2014b; Ioppolo et al. 2014), so their mobility should be strongly reduced compared to any other molecules of similar range of volatility (such as $\mathrm{CO}$ ). Figure 1, for the sake of simplicity, shows only the case in which NO dimers are unable to diffuse, which is an oversimplification. However, the important point here is the following: do $\mathrm{O}$ or $\mathrm{D}$ have to cross a water layer to react with NO (i.e., need bulk diffusion)?

The details of TPD profiles can help to discuss these possible locations: outer surface, inner surface, or bulk. In Fig. 7 panel a presents the TPD traces of mass 30 (in red) and mass 46 (in green) after the oxidation of $\mathrm{NO}$ without a water overlayer. As we have shown in Minissale et al. (2013c), TPD desorption curves of $\mathrm{NO}_{2}$ ices are characterized by two peaks at masses 30 and 46 (with a constant ratio $\mathrm{m} 46 / \mathrm{m} 30 \sim 13 \%$ ) at around $125 \mathrm{~K}$. We stress that only the presence of both mass 30 and 46 represents the desorption of $\mathrm{NO}_{2}$. In panels $b$ and $c$ we can see three peaks at mass 30 , named $\mathrm{A}, \mathrm{B}$, and $\mathrm{C}$ for the sake of clarity. The peak $A$ at $125 \mathrm{~K}$ is related to the desorption of surface $\mathrm{NO}_{2}$ since both masses 30 and 46 are present. Peak B at $148 \mathrm{~K}$ has no mass 46 components and it is therefore due to the desorption of NO. It is indeed the NO unreacted that is released during the rearrangement of the ASW, prior to the crystallization. This peak corresponds to $\mathrm{NO}_{\mathrm{b}}$ in water bulk (or inaccessible pores). Peak $\mathrm{C}$ shows masses 30 and 46 and it corresponds to the release of $\mathrm{NO}_{2}$ trapped in the pores of the water. The origin of this peak can be either $\mathrm{NO}_{\mathrm{b}}$ or $\mathrm{NO}_{\mathrm{s}}$ trapped during the reconstruction of the ice during the TPD. On panel d we plot the shape of the water desorption. It shows a two-peaks- desorption. The first peak corresponds to desorption of ASW. The ASW desorption is reduced when the crystallization occurs. The second peak corresponds to desorption of crystalline water (Smith et al. 1997). We can see that the NO desorption (peak B) occurs during the ASW desorption, whereas the $\mathrm{NO}_{2}$ desorption exactly matches with the water crystallization. This is known as the so-called volcano effect (May et al. 2013); when the water crystal is expanding, it expels all the impurities, which are $\mathrm{NO}_{2}$ in our present case. From panels a to $d$ in Fig. 7, we note a decrease of the peak at A; it is no longer visible in panel $\mathrm{d}$, meaning that all $\mathrm{NO}_{2}$ is formed from NO trapped inside the ice.

We now return to the different scenarios of NO reactivity depending on their initial location. $\mathrm{NO}_{2}$ desorbing from peak

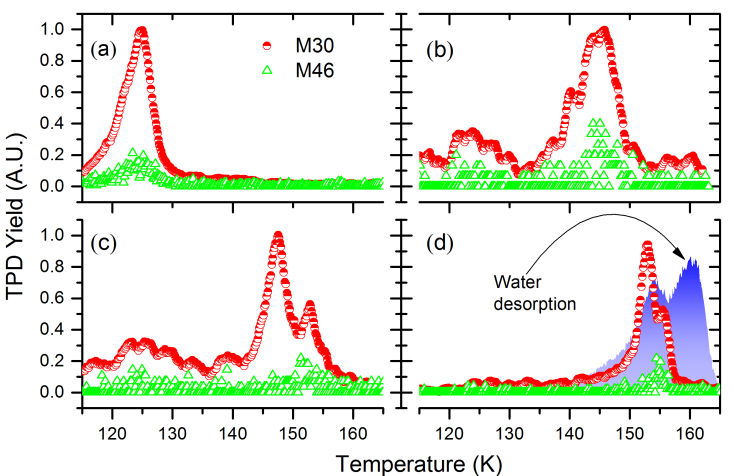

Fig. 7. Curves of TPD at mass 30 and 46 after O-exposure of $\mathrm{NO} / \mathrm{H}_{2} \mathrm{O}$ ice for different thickness of water ices held at $40 \mathrm{~K}$. Panel $a$ : $0 \mathrm{~mL}$. Panel b: $3 \mathrm{~mL}$. Panel c: $6 \mathrm{~mL}$. Panel d: $10 \mathrm{~mL}$.

A comes from the surface or from an open pore. The $\mathrm{C}$ peak indicates an origin within the volume, in bulk, or in open pores. We can clearly see the change in the balance from peak A to $\mathrm{C}$, corresponding to more and more NO that reacted in the pores or in the bulk. The increase of the B peak corresponds to the unreacted NO, which perfectly matches the plateau values observed in the IR spectra. The TPD traces show that the thicker the ice, the higher the proportion of reaction inside the pores. This was not certain before the study. It is possible to have imagined that the NO film would remain at the surface because it is completely repelled by water growth (water molecules impinging with the kinetic energy of room temperature) or segregated forming islands. Moreover, it seems that NO diffusion during the growth of water ice over layer is not efficient. This could be owing to the ability of NO to form dimers (Minissale et al. 2014b) that may have a low diffusion rate.

\subsection{Deuteration of NO ices}

In this section we describe experimental results of deuteration of NO. As studied by Congiu et al. (2012), the deuteration of NO brings to the formation of deuterated hydroxilamine $\left(\mathrm{ND}_{2} \mathrm{OD}\right)$ via the three subsequent $\mathrm{D}$-atom additions to nitrogen monoxide. Side reactions may lead to some $\mathrm{N}_{2} \mathrm{O}$ formation. The first step (NO consumption) is barrierless. In this case, we exposed $\mathrm{NO}$ ices held at $10 \mathrm{~K}$ to different amount of $\mathrm{D}$ atoms (up to $2.1 \mathrm{~mL}$ ). We studied three different chemical kinetics by varying the amount of water ice deposited on top of NO, i.e., 0 , 3 , and $6 \mathrm{~mL}$ of porous water ice. As in the case of $\mathrm{NO}+\mathrm{O}$ ice, we can easily follow the kinetics of NO consumption by looking to the decrease of IR band at $1770 \mathrm{~cm}^{-1}$. Unlike the $\mathrm{NO}+\mathrm{O}$ case, we cannot follow the kinetics of reaction products $\left(\mathrm{ND}_{2} \mathrm{OD}\right)$ since their IR signatures are hidden by the noise. Nevertheless by performing TPD experiments, we checked the presence of deuterated hydroxilamine after each experiment. NO kinetics is shown in Fig. 8; NO consumption reaches a plateau after a D-fluence of $\sim 1 \mathrm{~mL}$ in the case of bare NO. This could be explained by a cover effect of NO by the newly formed hydroxilamine: at very beginning of experiments, NO can react efficiently with D through Eley-Rideal mechanism; once hydroxilamine starts to form on the surface, it can partially cover NO, and $\mathrm{D}$ atoms have to diffuse to react with it. This means that surface density of $\mathrm{D}$ atoms increases and facilitates $\mathrm{D}_{2}$ formation through $\mathrm{D}+\mathrm{D}$ reaction. Somehow hydroxilamine could play the same role of water ice preventing a full NO consumption. The role of water ice is clearly visible for the experimental data 


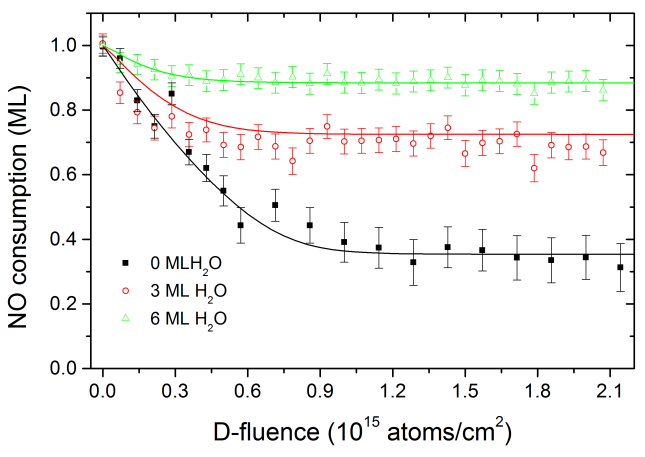

Fig. 8. Comparison between model (solid lines) and experimental data (dots). Experimental data are obtained by integrating IR peak of NO following to different fluences of $\mathrm{D}$ atoms for three different thickness of water ices $(0,3$, and $6 \mathrm{~mL})$ deposited on top of $\mathrm{NO}$ held at $40 \mathrm{~K}$. Simulated curves are obtained by using the $\alpha_{0}$ law for a deuterium bulk diffusion of $280 \mathrm{~K}$.

plotted in Fig. 8. When $3 \mathrm{~mL}$ of $\mathrm{H}_{2} \mathrm{O}$ are deposited, $0.3 \mathrm{~mL}$ of $\mathrm{NO}$ are consumed, while only $0.1 \mathrm{~mL}$ of NO reacts when $6 \mathrm{~mL}$ of $\mathrm{H}_{2} \mathrm{O}$ are deposited on top of it.

If we now compare the amount of NO unreacted (in $\mathrm{mL}$ ) for the $\mathrm{O}$ and $\mathrm{D}$ experiments, we find that it is $0.67 \pm 0.09 \mathrm{~mL}$ (for D) and $0.74 \pm 0.04 \mathrm{~mL}$ (for $\mathrm{O}$ ) for the case of $3 \mathrm{~mL}$ of water overlayer and $0.88 \pm 0.07$ and $0.89 \pm 0.02 \mathrm{~mL}$ for $6 \mathrm{~mL}$ of water. This amount is equal within our experimental error bars. We can conclude at this stage that the accessibility to NO for $\mathrm{O}$ atoms and $\mathrm{D}$ atoms seems to be identical.

\section{Model and discussion}

The model used to fit our experimental data is very similar to that described in Minissale et al. (2013c, 2015). In the case of NO oxygenation we follow the density of six chemical species: $\mathrm{O}$-surface atoms $\left(\mathrm{O}_{\mathrm{s}}\right)$, coming exclusively from the beam and diffusing only on the surface; O-bulk atoms $\left(\mathrm{O}_{\mathrm{b}}\right)$, reacting and diffusing only in the ice bulk; NO-surface molecules $\left(\mathrm{NO}_{\mathrm{s}}\right)$, deposited on the surface and reacting only with $\mathrm{O}_{\mathrm{s}}$; NO-bulk molecules $\left(\mathrm{NO}_{\mathrm{b}}\right)$, deposited on the surface and reacting only with $\mathrm{O}_{\mathrm{b}} ; \mathrm{NO}_{2}$ formed via $\mathrm{NO}_{\mathrm{s}}+\mathrm{O}_{\mathrm{s}}$ or $\mathrm{NO}_{\mathrm{b}}+\mathrm{O}_{\mathrm{b}}$; and finally $\mathrm{O}_{2}$, coming both from the beam and formed by solid-state reactions. Each differential equation is composed of different terms:

- positive terms contribute to the increase of species amount, i.e., a molecule (or atom) arrives from the gas phase or it is formed on the surface;

- negative terms indicate a decrease of species amount, i.e., a molecule (or atom) is consumed on the surface or it desorbs and returns to the gas phase.

The terms involving the Eley-Rideal (ER; gas-surface reactions) and Langmuir-Hinshelwood (LH) mechanisms (reaction induced by surface diffusion) are independent. For this reason we can determine the amount of a species formed (or consumed) via the ER or the LH mechanism. Below we show the set of differential equations built to simulate $\mathrm{NO}$ consumption and $\mathrm{NO}_{2}$ formation:

$$
\begin{aligned}
\mathrm{O}_{\mathrm{s}}^{\prime}(\mathrm{mL})= & \phi_{\mathrm{O}}\left(1-2 \mathrm{O}_{\mathrm{s}}-\mathrm{O}_{2}\right)-\phi_{\mathrm{O}_{2}} \mathrm{O}_{\mathrm{s}}-4 k_{\mathrm{O}_{\mathrm{s}}, \text { Dif }} \mathrm{O}_{\mathrm{s}} \mathrm{O}_{\mathrm{s}} \\
& -k_{\mathrm{O}_{\mathrm{s}}, \text { Dif }} \mathrm{O}_{\mathrm{s}} \mathrm{O}_{2}-\phi_{\mathrm{O}} \mathrm{NO}_{\mathrm{s}}-k_{\mathrm{O}_{\mathrm{s}}, \text { Dif }} \mathrm{O}_{\mathrm{s}} \mathrm{NO}_{\mathrm{s}} \\
& +k_{\mathrm{ObDif}}\left(\mathrm{O}_{\mathrm{b}}-\mathrm{O}_{\mathrm{s}}\right)-\mathrm{O}_{\mathrm{s}} \text { Des }_{\mathrm{O}_{\mathrm{s}}}, \\
\mathrm{O}_{\mathrm{b}}^{\prime}(\mathrm{mL})= & -k_{\mathrm{O}_{\mathrm{b}}, \text { Dif }}\left(\mathrm{O}_{\mathrm{b}}-\mathrm{O}_{\mathrm{s}}\right)-k_{\mathrm{O}_{\mathrm{b}}, \text { Dif }} \mathrm{O}_{\mathrm{b}} \mathrm{NO}_{\mathrm{b}}, \\
\mathrm{O}_{2}^{\prime}(\mathrm{mL})= & \phi_{\mathrm{O}_{2}}\left(1-\mathrm{O}_{\mathrm{s}}\right)-\phi_{\mathrm{O}} \mathrm{O}_{2}+\phi_{\mathrm{O}} \mathrm{O}_{\mathrm{s}} \\
& +2 k_{\mathrm{O}_{\mathrm{s}}, \text { Dif }} \mathrm{O}_{\mathrm{s}} \mathrm{O}_{\mathrm{s}}-k_{\mathrm{O}_{\mathrm{s}}, \text { Dif }} \mathrm{O}_{\mathrm{s}} \mathrm{O}_{2}-\mathrm{O}_{2} \operatorname{Des}_{\mathrm{O}_{2}},
\end{aligned}
$$

$$
\begin{aligned}
& \mathrm{NO}_{\mathrm{s}}^{\prime}(\mathrm{mL})=-\phi_{\mathrm{O}} \mathrm{NO}_{\mathrm{s}}-k_{\mathrm{O}_{\mathrm{s}}, \text { Dif }} \mathrm{O}_{\mathrm{s}} \mathrm{NO}_{\mathrm{s}}-\mathrm{NO}_{\mathrm{s}} \operatorname{Des}_{\mathrm{NO}_{\mathrm{s}}}, \\
& \mathrm{NO}_{b}^{\prime}(\mathrm{mL})=-k_{\mathrm{O}_{\mathrm{b}}, \text { Dif }} \mathrm{O}_{\mathrm{b}} \mathrm{NO}_{\mathrm{b}}, \\
& \mathrm{NO}_{2}^{\prime}(\mathrm{mL})=\phi_{\mathrm{O}} \mathrm{NO}_{\mathrm{s}}+k_{\mathrm{O}_{\mathrm{s}}, \text { Dif }} \mathrm{O}_{\mathrm{s}} \mathrm{NO}_{\mathrm{s}}+k_{\mathrm{O}_{\mathrm{b}} \text { Dif }} \mathrm{O}_{\mathrm{b}} \mathrm{NO}_{\mathrm{b}} .
\end{aligned}
$$

$\mathrm{O}_{\mathrm{s}}, \mathrm{O}_{\mathrm{b}}, \mathrm{O}_{2}, \mathrm{NO}_{\mathrm{s}}, \mathrm{NO}_{\mathrm{b}}$, and $\mathrm{NO}_{2}$ are the densities (expressed in fractions of $\mathrm{mL}$ ) of the different species, $\phi_{\mathrm{O}}$ is the flux of $\mathrm{O}$ atoms, and $\phi_{\mathrm{O}_{2}}$ is the nondissociated fraction of $\mathrm{O}_{2}$ defined in Sect. 2. We define

$$
\begin{aligned}
& \operatorname{Des}_{\mathrm{O}_{\mathrm{s}}}=v e^{\frac{-E_{\mathrm{O}_{\mathrm{s}} \text {,des }}}{T}}, \text { with } E_{\mathrm{O}_{\mathrm{s}}, \text { des }}=1400 \mathrm{~K} \\
& \operatorname{Des}_{\mathrm{O}_{2}}=v e^{\frac{-E_{\mathrm{O}_{2}, \text { des }}}{T}}, \text { with } E_{\mathrm{O}_{2}, \text { des }}=1100 \mathrm{~K} \\
& \operatorname{Des}_{\mathrm{NO}}=v e^{\frac{-E_{\mathrm{NO}, \text { des }}}{T}}, \text { with } E_{\mathrm{NO}, \mathrm{des}}=1300 \mathrm{~K} \\
& k_{\mathrm{O}_{\mathrm{s}}, \text { Dif }}=v e^{\frac{-E_{\mathrm{O}_{\mathrm{s}} \text {,dif }}}{T}} \text {, with } E_{\mathrm{O}_{\mathrm{s}}, \text { dif }}=750 \mathrm{~K} \\
& k_{\mathrm{O}_{\mathrm{b}}, \text { Dif }}=v e^{\frac{-E_{\mathrm{O}_{\mathrm{b}} \text {,dif }}}{T}}, \text { with } E_{\mathrm{O}_{\mathrm{b}}, \text { dif }} \text { a free parameter, }
\end{aligned}
$$

where $T$ is the sample temperature and $v=10^{12} \mathrm{~s}^{-1}$ is the trial

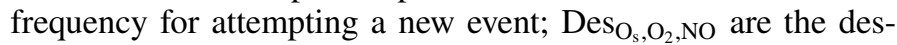
orption probabilities of $\mathrm{O}_{\mathrm{s}}, \mathrm{O}_{2}$, and $\mathrm{NO}$, respectively; $k_{\mathrm{O}_{\mathrm{s}} \text {,dif }}$ and $k_{\mathrm{O}_{\mathrm{b}} \text {,dif }}$ are the thermal diffusion probability of $\mathrm{O}$ atoms on surface (s) and in the bulk (b). We point out that for the diffusion probability we only consider a pure thermal diffusion barrier since quantum effects are negligible at high temperatures $(>30 \mathrm{~K})$. We stress that the only free parameters are $E_{\mathrm{O}_{\mathrm{b}} \text {,dif }}$ and the initial amounts of $\mathrm{NO}_{\mathrm{s}}$ and $\mathrm{NO}_{\mathrm{b}}$.

As described before water ice is deposited on top of NO molecules. Depending on the amount of water ice deposited, $\mathrm{NO}$ molecules can be accessible by bulk $\left(\mathrm{NO}_{\mathrm{b}}\right)$ or surface $\left(\mathrm{NO}_{\mathrm{s}}\right)$ paths. We use a single parameter, called $\alpha$, to take into account the initial amount of $\mathrm{NO}_{\mathrm{b}}(\alpha)$ and $\mathrm{NO}_{\mathrm{s}}(1-\alpha)$ molecules.

The main difficulty of the analysis lies is the fact that we do not know the amount of NO initially present in the bulk $\left(\mathrm{NO}_{\mathrm{b}}\right)$. The consumption of $\mathrm{NO}$ can occur via surface reactions of $\mathrm{NO}_{\mathrm{s}}$ or via diffusion through the ice. We have a unique observable NO, but two competing and potentially compensating pathways. Actually we can obtain the same level of consumption if we increase both $\alpha$ and bulk diffusion. In other words, more NO can be initially covered if the bulk diffusion is more efficient. Therefore, we need to explore the whole domain of bulk diffusion and initial $\alpha$ coefficient to simulate the full kinetic of NO consumption.

Let us first focus on the possible values of $\alpha\left(\mathrm{NO}_{\mathrm{b}}\right)$. The amounts of $\mathrm{NO}_{\mathrm{b}}$ and $\mathrm{NO}_{\mathrm{s}}$ are related by the obvious closure relation $\mathrm{NO}=\mathrm{NO}_{\mathrm{b}}+\mathrm{NO}_{\mathrm{s}}$. Because $\mathrm{NO}=1(1 \mathrm{~mL}$ of $\mathrm{NO}$ is initially deposited $)$ in all our experiments, $\mathrm{NO}_{\mathrm{s}}=(1-\alpha)$. At the present stage, we ignore the dependency of $\alpha$ with the thickness of ASW. By definition $\alpha=0$ when there is no water deposition. We can suppose that $\alpha$ is monotonically increasing with the water ice layers (WIL) and that $\alpha$ cannot be larger than the WIL. Here we suppose that at least one water molecule is required to occupy one adsorption site. The value $\alpha$ tends toward 1 when the ASW thickness tends to infinity.

Moreover we know that the $\mathrm{NO}+\mathrm{O}$ reaction is a barrierless reaction and so all the NO accessible via surface paths should react. This means that the amount of $\mathrm{NO}_{\mathrm{s}}$ (i.e., 1- $\alpha$ ) cannot be greater than the amount of NO reacted. This hypothesis is reinforced by the fact that in all our experiments, we reach a plateau, indicating that all the possible reactants have been consumed. In Fig. 9 we plot in blue dots the amount of NO unreacted as a function of WILs deposited on top of the NO layer. The possible values of $\alpha$ are represented by the colored zone. The values of 


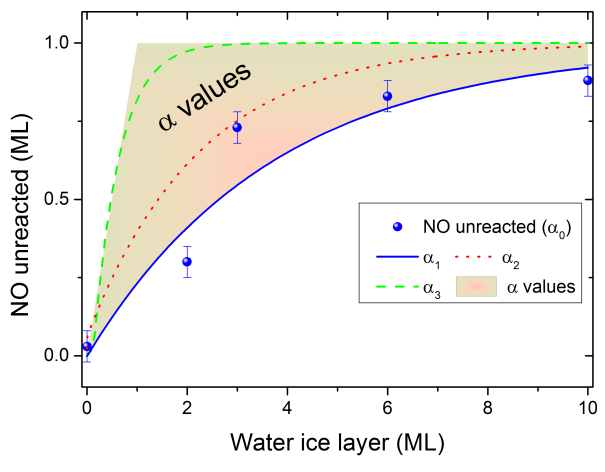

Fig. 9. Colored zone: possible values of NO located inside the bulk of ice $\left(\mathrm{NO}_{\mathrm{b}}=\alpha\right)$ as a function of the WIL thickness. Blue points: measured amounts of unreacted NO. Blue, red, and green lines represent three possible parametrized repartitions of initially covered NO molecules

$\alpha$, whatever is the WIL are comprised of between 0 for WIL $=0$ and 1 for $\mathrm{WIL}=\infty$. Moreover, taking into account the previous remark that $\mathrm{NO}_{\mathrm{s}}$ (i.e., 1- $\alpha$ ) cannot be greater than the amount of NO reacted, therefore $\alpha$ is necessarily greater than the unreacted NO. In other words, the contribution of bulk diffusion cannot be negative. So the $\alpha$ domain is restricted to the portion of the Fig. 9 above the experimental values $\left(\alpha_{0}\right)$. To materialize the inferior limit of the possible $\alpha$, we took an exponential saturation law $\alpha(\mathrm{WIL})=1-e^{-\frac{\mathrm{WIL}}{P_{1}}}$ where $P_{1}$ is the fitting parameters. The curve $\alpha_{1}$ (WIL) is represented in blue in Fig. 9 and it corresponds to the situation of a low number of NO initially present in the bulk, $\alpha_{2}$ (red dotted curve) to an intermediate number of initial $\mathrm{NO}_{\mathrm{b}}$, and $\alpha_{3}$ (green dashed curve) represents the case where NO molecules are perfectly covered with water ice, and therefore the reactivity can only proceed through bulk diffusion. The three laws are given by

$\alpha_{1}(\mathrm{WIL})=1-e^{-\frac{\mathrm{WL}}{3.447}}$,

$\alpha_{2}(\mathrm{WIL})=1-e^{-\frac{\mathrm{WL}}{2.247}}$,

$\alpha_{3}(\mathrm{WIL})=1-e^{-\frac{\mathrm{WL}}{0.515}}$.

We stress that these are empiric laws that have been defined to explore the space parameter of the possible $\alpha$ values as we can see in Fig. 9.

Figure 10 presents typical comparison of our model and the experiments. We use the kinetic of the NO consumption (and $\mathrm{NO}_{2}$ formation) for different $\mathrm{O}$ fluences. We can very satisfactorily reproduce our experimental data using the $\alpha_{0}$ values, which are indeed the values of $\alpha$ equal to the unreacted NO obtained from the plateaus observed in IR spectra. In this case, the best fit is obtained for any value of bulk diffusion greater than $950 \mathrm{~K}$ as can be seen on the right panel of the Fig. 10. This value is to be compared with the value of surface diffusion taken as $750 \mathrm{~K}$. It corresponds to a ratio of $\sim 150$ of the two diffusion rates.

Other scenarios of initial covering of NO are tested using different $\alpha_{x}$ laws. We can see on the $\chi^{2}$ graph that there exists a minimum for which it is possible to compensate an initial coverage with a lower diffusion barrier. These minima have higher absolute values with the increase of the initial NO coverage (from 1 to 3), and logically show values with lower and lower diffusion barriers, in line with what is expected. The values of $\chi^{2}$ are higher but, taking into account that the $\alpha_{0}$ has the best possible absolute values of $\chi^{2}$ because it is made from the right plateau values, it is not a strong reason to reject these possibilities of initial coverage. We can for example argue that our $\alpha_{x}$ laws are not exactly representative of the initial coverages and that another repartition would probably get a lower, and therefore better, minimal value of $\chi^{2}$. However, on the right side of Fig. 10 we can see that the best fits correspond to unrealistic situations. In particular, the values predicted at high fluences are always decreasing and therefore there is no plateau, as observed in experimental data. Therefore, the best solution is the case of $\alpha_{0}$, which corresponds the scenario of the minimum of NO initially covered with the water ice, and thus to a slow bulk diffusion, which needs to be at least two order of magnitude slower than the surface diffusion.

We also built a similar model to simulate the NO $+\mathrm{D}$ kinetics. We considered eight species $\mathrm{NO}_{\mathrm{s}}, \mathrm{NO}_{\mathrm{b}}, \mathrm{D}_{\mathrm{s}}, \mathrm{D}_{\mathrm{b}}, \mathrm{D}_{2}, \mathrm{NOD}$, $\mathrm{NDOD}$, and $\mathrm{N}_{2} \mathrm{OD}$. The desorption energy of $\mathrm{D}$ and $\mathrm{D}_{2}$ was fixed to $600 \mathrm{~K}$ and the diffusion energy for $\mathrm{D}_{\mathrm{s}}$ to $210 \mathrm{~K}$ (Wakelam et al. 2017). Even in this case we considered three laws for $\alpha$ and different values of bulk diffusion. We obtained results similar to the case of NO oxygenation. The best fit, shown in Fig. 8, has been found for the $\alpha_{0}$ law, $\left(\mathrm{NO}_{\mathrm{s}}=\mathrm{NO}_{\text {reacted }}\right)$ and the minimum value of bulk diffusion is $E_{\text {Dbdif }}=280 \mathrm{~K}$. In this case, the ratio of surface diffusion rates to bulk diffusion rates is larger than 1000 .

In both cases, NO oxygenation and deuteration, the chemistry seems to be driven by surface reactions (including the pores). Lowering the value of $E_{\text {bdif }}$ to values closer to $E_{\text {sdif }}$ induces a faster kinetics, that would slowly consumed the $\mathrm{NO}_{\mathrm{b}}$, which is not observed experimentally. A good agreement between experimental and simulation results can be found using

\section{$\frac{E_{\text {bulk-diffusion }}}{E_{\text {surface-diffusion }}} \geq 1.3$.}

\section{Astrophysical implications and conclusions}

In this work, we present an experimental comparison of the bulk diffusion (i.e., the crossing of a compact/closed ice layer) and surface diffusion (including in the pores) of oxygen and deuterium that can occur in conditions mimicking the interstellar ices. In particular we evaluated the ratio of surface to bulk diffusion, where the surface diffusion is the diffusion occurring on the top surface and on the surface of the (open) pores. Potentially surface diffusion can take place in the closed pores too, but it can be neglected as a first approximation since it has to be triggered by bulk diffusion.

We use NO as a chemical tracer. Under O or D exposure, NO is rapidly consumed, down to a plateau indicating that NO can no longer be reached by atoms in our experimental conditions. To mimic the initial fast consumption and the following plateau, only a bulk diffusion (through water ice) two orders of magnitude slower than surface diffusion for $\mathrm{O}$ and three orders of magnitude for D can fit our data. We found a lower limit for bulk diffusion of 950 and $280 \mathrm{~K}$ for $\mathrm{O}$ and $\mathrm{D}$, respectively, and a $E_{\mathrm{diff} \text { bulk }} / E_{\mathrm{diff} \text { surf }} \geq 1.3$ in both cases. We stress that we did not perform experiments using $\mathrm{H}$ atom. Since the diffusion of $\mathrm{H}$ could be different from $\mathrm{D}$, the conclusion regarding $\mathrm{D}$ atoms could not be totally valid for $\mathrm{H}$ atoms. In the interstellar medium, the existence of ice mantle is ubiquitous as soon as visual extinction reaches a value of 3 . This corresponds to regions where density raises to $\sim 10^{3-4} \mathrm{~mol} \mathrm{~cm}{ }^{-3}$. In these conditions, one layer of the mantle takes about $1000 \mathrm{yr}$ to build up for a total of $10^{5} \mathrm{yr}$ to reach a 100-layer thick mantle. After this point, most of the gas species should already have accreted on grains, with few notable exceptions such as $\mathrm{H}, \mathrm{He}, \mathrm{H}_{2}$, and some of close related species 

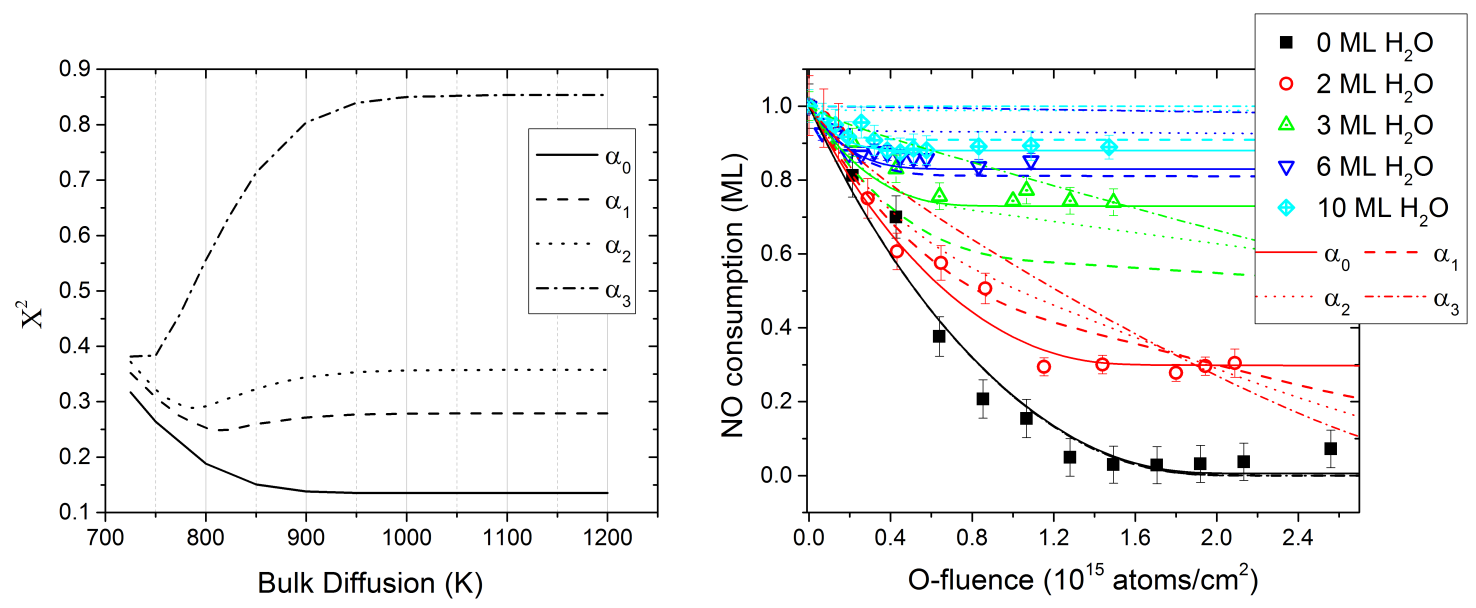

Fig. 10. Left panel: $\chi^{2}$ values comparing model and data for different $\alpha$ laws as a function of bulk diffusion energy. The value $\alpha_{0}$ corresponds to the exact experimental values of unreacted species. Right panel: comparison between model (lines) and experimental data (dots). Experimental data are obtained by integrating IR peaks of $\mathrm{NO}$ and $\mathrm{NO}_{2}$ as a function of O-atom fluence for five different thickness of water ices $(0,2,3,6$, and $10 \mathrm{~mL}$ ) held at $40 \mathrm{~K}$. Simulated curves are obtained by using three laws for the $\alpha$ parameter $\left(\alpha_{1}, \alpha_{2}\right.$, and $\left.\alpha_{3}\right)$ and measured experimental values $\left(\alpha_{0}\right)$ for the best case corresponding to the minimum $\chi^{2}$ of left panel. For $\alpha_{0}$ any choice of diffusion parameter greater than $950 \mathrm{~K}$ gives similar results.

such as $\mathrm{H}_{3}^{+}$and $\mathrm{N}_{2} \mathrm{H}^{+}$. This is why interstellar ices enter a second stage of their chemical evolution, whose duration depends on the mass of the to-be-born star, before ongoing processes linked to the more energetic next phases of star formation. During the growth of the mantle, one $\mathrm{H}$ atom lands on grains every $\sim 2 \times 10^{4}$ s (see Fig. 8 of Congiu et al. 2014). It can completely scan the surface of a grain (radius $\simeq 0.1 \mu \mathrm{m}$ ) in about $10^{3} \mathrm{~s}$. In the case of $\mathrm{D}$ atoms, the lower limit of the bulk diffusion $(280 \mathrm{~K})$ corresponds to a life time on the surface of $\sim 1 \mathrm{~s}$ at $10 \mathrm{~K}\left(\tau=k_{\mathrm{D}_{\mathrm{b}} \text {,Dif }}^{-1}\right)$. It means that $\mathrm{H}$ atoms can scan the surface before penetrating the bulk of the ice, but it could penetrate the ice before the arrival of another $\mathrm{H}$ atom. This implies that the $\mathrm{H}$ diffusion through the bulk of the ice cannot be fully ruled out with our lower limit of $\mathrm{H}$ bulk diffusion. Only a factor of more than $10^{6}$ between the surface and bulk diffusion rates would prevent bulk penetration, the latter being too slow to compete with the $\mathrm{H}$ accretion of the gas phase, and its subsequent transformation into $\mathrm{H}_{2}$. However, since the surface diffusion rate is still at least 1000 times faster than the bulk diffusion, any surface reactant with a concentration greater than a thousandth would statistically react with the $\mathrm{H}$ atoms. In other words, $\mathrm{H}$ could penetrate deeper in the ice mantle only if very few reactants are present on the outer surface of grains. Thus, we conclude that the surface hydrogenation, in our experiments as well as in the interstellar medium, is dominant over bulk diffusion, which cannot be totally excluded only on the basis of a direct extrapolation of our experiments. In such considerations, we neglect isotopic effects that are probably important but hard to take into account since both surface and bulk diffusions should be affected. For $\mathrm{O}$ atoms, crossing the first water bulk layer should take $\sim 10^{29} \mathrm{~s}$, which means that it is not possible (within the frame of the classical formalism we use).

However, we point out our values are lower limits for the bulk diffusion barriers as shown by the flat shape of the $\chi^{2}$ curve of Fig. 10. The present experiments do not show directly whether $\mathrm{H}$ diffusion through the ice bulk can reduce the lifetime of radicals formed inside the volume of the ice mantle during UV irradiation or during other energetic processes. The presence of radicals during the initial building of the ice mantle is probably excluded thanks to the high mobility of $\mathrm{H}$ atoms at any temperature (Senevirathne et al. 2017). Our experiments show that the only very first layers of the mantle are reacting and models considering that surface reactions rule the ice building (e.g., Vasyunin et al. 2017) seem thus coherent with our laboratory experiments. Hydrogenation or oxidation experiments (Hiraoka et al. 2002; Watanabe et al. 2004; Fuchs et al. 2009; Jing et al. 2011; Ward \& Price 2011) have used similar or greater atomic fluxes. This means in a first approximation that the surface to bulk diffusion efficiency is the same or greater. Actually, the higher the flux, the higher the self reactivity, the lower the possibility to penetrate inside the molecular substrate. With the present study, we can understand why, by construction, laboratory experiments favor surface instead of bulk reactivity. However, we provide a lower limit confirming that surface diffusion of $\mathrm{H}$ is an order of magnitude faster than bulk diffusion through the ice, even though it certainly changes with the type of molecular layer used.

Finally some works suggest the existence of an efficient ice segregation mechanism of both interstellar (Öberg et al. 2011; Allodi et al. 2014) and cometary ices (Dello Russo et al. 2011; McKay et al. 2018). Nevertheless ice segregation is still an open question in astrochemistry (Boogert et al. 2015). The possible $\mathrm{H}$ atoms migration inside the bulk can strongly change the chemical composition in the whole ice thickness, but simple thermal differentiation could also be the reason for the observed segregation. This aspect could now be tested thanks to Monte Carlo simulations (Cazaux et al. 2010; Cuppen et al. 2017) using our boundary limits of bulk diffusion barriers.

Acknowledgements. T.N. is a Ph.D. student granted by the Labex MICHEM program. This work was supported by the Program DIM-ACAV, and now DIM$\mathrm{ACAV}+$, of the Region Ile de France, the CNRS - INSU program Physique et Chimie du Milieu Interstellaire (PCMI), supported by the CNES.

\section{References}

Accolla, M., Congiu, E., Dulieu, F., et al. 2011, Phys. Chem. Chem. Phys., 13, 8037

Accolla, M., Congiu, E., Manicò, G., et al. 2013, MNRAS, 429, 3200

Allodi, M. A., Ioppolo, S., Kelley, M. J., McGuire, B. A., \& Blake, G. A. 2014, Phys. Chem. Chem. Phys., 16, 3442 
Amiaud, L., Dulieu, F., Fillion, J.-H., Momeni, A., \& Lemaire, J. L. 2007, J. Chem. Phys., 127, 144709

Anders, E., Hayatsu, R., \& Studier, M. H. 1974, ApJ, 192, L101

Boogert, A. A., Gerakines, P. A., \& Whittet, D. C. 2015, ARA\&A, 53, 541

Caselli, P., \& Ceccarelli, C. 2012, A\&ARv, 20, 56

Cazaux, S., Cobut, V., Marseille, M., Spaans, M., \& Caselli, P. 2010, A\&A, 522, A74

Ceccarelli, C., Caselli, P., Fontani, F., et al. 2017, ApJ, 850

Chaabouni, H., Bergeron, H., Baouche, S., et al. 2012, A\&A, 538, A128

Congiu, E., Matar, E., Kristensen, L. E., Dulieu, F., \& Lemaire, J. L. 2009, MNRAS, 397, L96

Congiu, E., Chaabouni, H., Laffon, C., et al. 2012, J. Chem. Phys., 137, 054713

Congiu, E., Minissale, M., Baouche, S., et al. 2014, Faraday Discuss., 168, 151

Cooke, I. R., Öberg, K. I., Fayolle, E. C., Peeler, Z., \& Bergner, J. B. 2018, ApJ, 852,75

Cuppen, H. M., Walsh, C., Lamberts, T., et al. 2017, Space Sci. Rev., 212, 1

Dello Russo, N., Vervack, Jr. R. J., Lisse, C. M., et al. 2011, ApJ, 734, L8

Fuchs, G. W., Cuppen, H. M., Ioppolo, S., et al. 2009, A\&A, 505, 629

Ghesquière, P., Ivlev, A., Noble, J. A., \& Theulé, P. 2018, A\&A, 614, A107

Hama, T., Kuwahata, K., Watanabe, N., et al. 2012, ApJ, 757, 185

He, J., Emtiaz, S. M., \& Vidali, G. 2017, ApJ, 837, 65

Hidaka, H., Kouchi, A., \& Watanabe, N. 2007, J. Chem. Phys., 126, 204707

Hiraoka, K., Sato, T., Sato, S., et al. 2002, ApJ, 577, 265

Ioppolo, S., Fedoseev, G., Minissale, M., et al. 2014, Phys. Chem. Chem. Phys., 16,8270

Jing, D., He, J., Brucato, J., et al. 2011, ApJ, 741, L9

King, D. A., \& Wells, M. G. 1972, Surf. Sci, 29, 454

Kristensen, L. E., van Dishoeck, E. F., van Kempen, T. A., et al. 2010, A\&A, 516, A57

Lauck, T., Karssemeijer, L., Shulenberger, K., et al. 2015, ApJ, 801, 118

Matar, E., Congiu, E., Dulieu, F., Momeni, A., \& Lemaire, J. L. 2008, A\&A, 492, L17

May, R. A., Smith, R. S., \& Kay, B. D. 2013, J. Chem. Phys., 138, 104501

McKay, A. J., Cochran, A. L., DiSanti, M. A., et al. 2018, Icarus, 309, 1

Minissale, M., Congiu, E., Baouche, S., et al. 2013a, Phys. Rev. Lett., 111, 05320

Minissale, M., Congiu, E., Baouche, S., et al. 2013b, Chem. Phys. Lett, 565, 52
Minissale, M., Congiu, E., Manicò, G., Pirronello, V., \& Dulieu, F. 2013c, A\&A, 559, A49

Minissale, M., Congiu, E., \& Dulieu, F. 2014a, J. Chem. Phys., 140, 074705

Minissale, M., Fedoseev, G., Congiu, E., et al. 2014b, Phys. Chem. Chem. Phys., 16,8257

Minissale, M., Loison, J.-C., Baouche, S., et al. 2015, A\&A, 577, A2

Minissale, M., Congiu, E., \& Dulieu, F. 2016, A\&A, 585, A146

Mispelaer, F., Theulé, P., Aouididi, H., et al. 2013, A\&A, 555, A13

Nguyen, T., Baouche, S., Congiu, E., et al. 2018, A\&A, 619, A111

Noble, J. A., Congiu, E., Dulieu, F., \& Fraser, H. J. 2012, MNRAS, 421, 768

Oba, Y., Miyauchi, N., Hidaka, H., et al. 2009, ApJ, 701, 464

Öberg, K. I., Fayolle, E. C., Cuppen, H. M., van Dishoeck, E. F., \& Linnartz, H. 2009, A\&A, 505, 183

Öberg, K. I., Boogert, A. C. A., Pontoppidan, K. M., et al. 2011, ApJ, 740, 109

Orzechowska, G. E., Goguen, J. D., Johnson, P. V., Tsapin, A., \& Kanik, I. 2007, Icarus, 187, 584

Palumbo, M. E. 2006, A\&A, 453, 903

Palumbo, M., Baratta, G., Leto, G., \& Strazzulla, G. 2010, J. Mol. Struct., 972, 64

Papoular, R. 2005, MNRAS, 626, 489

Pirronello, V., Liu, C., Roser, J. E., \& Vidali, G. 1999, A\&A, 344, 681

Rimola, A., Taquet, V., Ugliengo, P., Balucani, N., \& Ceccarelli, C. 2014, A\&A, 572, A70

Senevirathne, B., Andersson, S., Dulieu, F., \& Nyman, G. 2017, Mol. Astrophys., 6,59

Smith, R. S., Huang, C., Wong, E. K. L., \& Kay, B. D. 1997, Phys. Rev. Lett., 79,909

Stevenson, K. P., Kimmel, G. A., Dohnálek, Z., Smith, R. S., \& Kay, B. D. 1999 Science, New Ser., 283, 1505

Tielens, A. G. G. M., \& Hagen, W. 1982, A\&A, 114, 245

Vasyunin, A., Caselli, P., Dulieu, F., \& Jiménez-Serra, I. 2017, ApJ, 842

Wakelam, V., Bron, E., Cazaux, S., et al. 2017, Mol. Astrophys., 9, 1

Ward, M. D., \& Price, S. D. 2011, ApJ, 741, 121

Watanabe, N., Nagaoka, A., Shiraki, T., \& Kouchi, A. 2004, ApJ, 616, 638

Watanabe, N., Kimura, Y., Kouchi, A., et al. 2010, ApJ, 714, L233 